\title{
Traumatismo grave del miembro superior relacionado con el uso de una escalera mecánica. Reporte de caso pediátrico
} Severe upper limb trauma related to escalator use. Pediatric case report

\author{
Dra. Guillermina Melendi ${ }^{a}$, Dr. Rodolfo P. Moreno ${ }^{a}$ Dr. Alberto Hernándeza ${ }^{a}$ Dra. Yanina Hernández ${ }^{a}$, \\ Dra. Cecilia Seligra ${ }^{a}$, Dr. Javier Fain ${ }^{a}$, Dr. Alejandro Grun ${ }^{b}$, Dr. Carlos E. Sereday ${ }^{c}$ y Dr. Gustavo Cardignia
}

\begin{abstract}
RESUMEN
Las lesiones relacionadas con escaleras mecánicas suelen ser poco frecuentes, pero pueden constituir una emergencia médica con complicaciones potencialmente peligrosas. Se describe el grave compromiso en el miembro superior relacionado con una lesión ocurrida en una escalera mecánica.

Paciente de 2 años, que sufrió una caída al bajar por una escalera mecánica, y terminó con el brazo izquierdo atrapado entre uno de los escalones y los peines del descenso del escalón terminal. Ingresó a Emergencias, donde, tras realizar las medidas de estabilización inicial, se trasladó a cirugía para el retiro del cuerpo extraño. No se encontró compromiso vascular o nervioso, pero sí pérdida grave de tejido celular subcutáneo. Se retiró el peine de metal, y se realizó la cirugía reparadora del miembro afectado. Requirió cuatro intervenciones más por Cirugía Plástica y Reparadora. La paciente tuvo buena evolución clínica y recibió el alta sin secuelas funcionales.

Palabras clave: escaleras mecánicas, heridas y traumatismos, niño.
\end{abstract}

\begin{abstract}
Escalator-related injuries are rare but can be a medical emergency with potentially dangerous complications. The severe upper limb involvement related to injury occurred on an escalator is described.

A two year-old patient suffered a fall going down an escalator; her left arm was caught between one of the steps and the comb of the last step.

She was admitted to the Emergency Room for the initial stabilization. The foreign body was removed in the operating room. No vascular or nervous compromise was found, but there was severe loss of subcutaneous cellular tissue. The metal comb was removed, and repair surgery was performed on the affected limb. She required four more surgical interventions for plastic and reconstructive surgery. The patient had a good clinical evolution and was discharged without functional sequelae. Key words: escalators, wounds and injuries, child.
\end{abstract}

a. Servicio de Pediatría, Sanatorio de la Trinidad Palermo.

b. Traumatología Infantil, Sanatorio de la Trinidad Palermo.

c. Cirugía Plástica y Reparadora, Sanatorio de la Trinidad Palermo.

Sanatorio de la Trinidad Palermo, Ciudad de Buenos Aires, Argentina.

Correspondencia:

Dr. Rodolfo P. Moreno: morenopablorod@hotmail.com

Financiamiento: Ninguno.

Conflicto de intereses: Ninguno que declarar.

Recibido: 6-8-2020

Aceptado: 18-12-2020 http: / / dx.doi.org/10.5546/ aap.2021.e256

Cómo citar: Melendi G, Moreno RP, Hernández A, Hernández Y, et al. Traumatismo grave del miembro superior relacionado con el uso de una escalera mecánica. Reporte de caso pediátricoo. Arch Argent Pediatr 2021;119(3):e256-e260.

\section{INTRODUCCIÓN}

La primera escalera mecánica fue patentada en 1892 e instalada en Nueva York como un paseo de diversión. ${ }^{1}$ Hoy se han convertido en un medio de transporte de uso común y constituyen una característica de los centros comerciales, las estaciones de subterráneos y/o trenes y grandes edificios. En los Estados Unidos de América, se informan, anualmente, alrededor de 10000 lesiones relacionadas con escaleras mecánicas en adultos que requieren tratamiento en Emergencias, ${ }^{2}$ pero existen muy pocas publicaciones en la literatura médica en cuanto a estas lesiones en pediatría.

Las lesiones en niños relacionadas con las escaleras mecánicas son poco frecuentes, pero pueden causar un amplio espectro de traumas, desde leves a otros muy graves, con pérdida de las manos o los pies, de todo el brazo o la pierna. ${ }^{3}$ A menudo, se asocian con resbalones, tropiezos o caídas. Los traumatismos en escaleras mecánicas son más frecuentes en los menores de cinco años ${ }^{4}$ y ocurren, en su mayoría, en las extremidades del niño. Se describe el grave compromiso del miembro superior relacionado con una lesión ocurrida en una escalera mecánica.

\section{CASO CLÍNICO}

Una paciente de 2 años de edad, mientras bajaba una escalera mecánica en un centro comercial, tomada de la mano de un adulto, sufrió una caída en dominó y terminó con el brazo izquierdo atrapado entre uno de los escalones y los peines del descenso del escalón terminal. La escalera se detuvo automáticamente $\mathrm{y}$, durante el rescate realizado por bomberos, fue necesario desarmarla, debido al ingreso profundo de los 
peines del escalón terminal de la escalera en el tejido celular subcutáneo del brazo.

Se trasladó al Servicio de Emergencias, con los peines incrustados en el antebrazo y el brazo izquierdo (Figuras 1 y 2). Ingresó lúcida, reactiva, irritable, con llanto inconsolable, sin sangrado activo evidente, pálida y sudorosa, con estabilidad hemodinámica. El miembro superior izquierdo se encontraba pálido, más frío en comparación con el derecho y con leve alteración del relleno capilar. Los pulsos estaban conservados y eran simétricos, sin signos de síndrome compartimental.

Se monitorizó en forma inmediata en el shock room pediátrico, con un índice shock de 1,6, saturación del $96 \%$ (fracción inspirada de oxígeno $-\mathrm{FiO}_{2}-:$ 0,21). Se indicó la reanimación con volumen, aporte de oxígeno y analgesia con opioides por vía endovenosa. La ecografía Doppler color mostró buen flujo arterial en ambos brazos.

Se interconsultó con Traumatología Infantil y con Cirugía Vascular, y se decidió realizar la evaluación bajo anestesia en quirófano. Se decidió no tomar radiografías en ese momento dada la presencia de los peines de la escalera y posponerlas para quirófano.

FIGURA 1. Imagen del brazo de la niña con la incrustación de los escalones de la escalera mecánica (fotografía tomada en quirófano bajo anestesia)

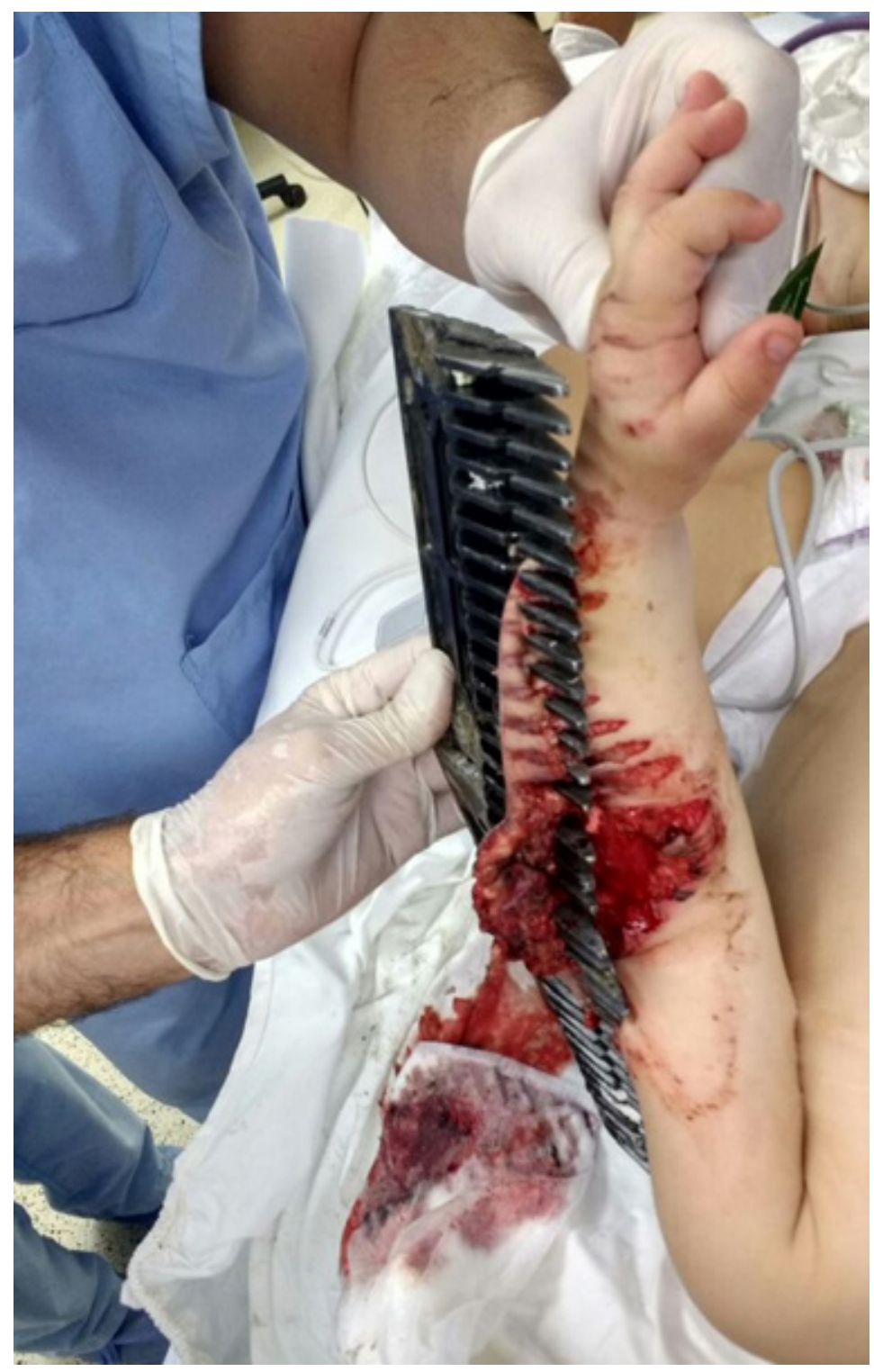


A la hora del ingreso, se sometió a cirugía, en una intervención conjunta de los equipos de Traumatología Infantil y Cirugía Vascular Periférica. No se encontró compromiso vascular ni nervioso, pero sí pérdida grave de tejido celular subcutáneo. Se retiró el peine de metal, y se realizó la cirugía reparadora del miembro afectado. Posteriormente, se internó en Terapia Intensiva Pediátrica.

El segundo ingreso a quirófano fue con el equipo quirúrgico de Cirugía Plástica y Reparadora, a las 24 horas de internación: plástica por colgajos, curación de la herida y colocación de sistema VAC-KCI ${ }^{\circledR}$ (Figura 3). Requirió, en total, cuatro ingresos posteriores a quirófano para la evaluación de los colgajos y la curación de la herida. No hubo complicaciones infecciosas.

La evolución clínica general y de la lesión en el miembro superior izquierdo fue muy buena. Egresó al octavo día de internación y se mantuvo en seguimiento ambulatorio con su pediatra de cabecera y el equipo de Cirugía Plástica y Reparadora.

\section{DISCUSIÓN}

Las lesiones relacionadas con escaleras mecánicas son poco frecuentes, pero pueden ocasionar graves traumatismos..$^{3,4}$ En el caso presentado, se destaca la adecuada evaluación inicial del efectivo del Cuerpo de Bomberos afectado al centro comercial, quien, al desarmar la escalera mecánica sin remover el elemento penetrante de la extremidad, impidió un sangrado masivo por los probables daños vasculares y locales de la zona afectada.

En los Estados Unidos de América, los Centers for Disease Control and Prevention (CDC) emitieron una alerta por este tema e instaron a la capacitación de los trabajadores de los lugares donde había ascensores y escaleras mecánicas. ${ }^{5}$ En la Ciudad de Buenos Aires, a partir del incendio del establecimiento República de Cromañón en 2004, la Dirección General de Planeamiento de Emergencias y Coordinación de Bomberos del Gobierno de la Ciudad de Buenos Aires dispuso el despliegue de sus efectivos en hospitales y centros comerciales.

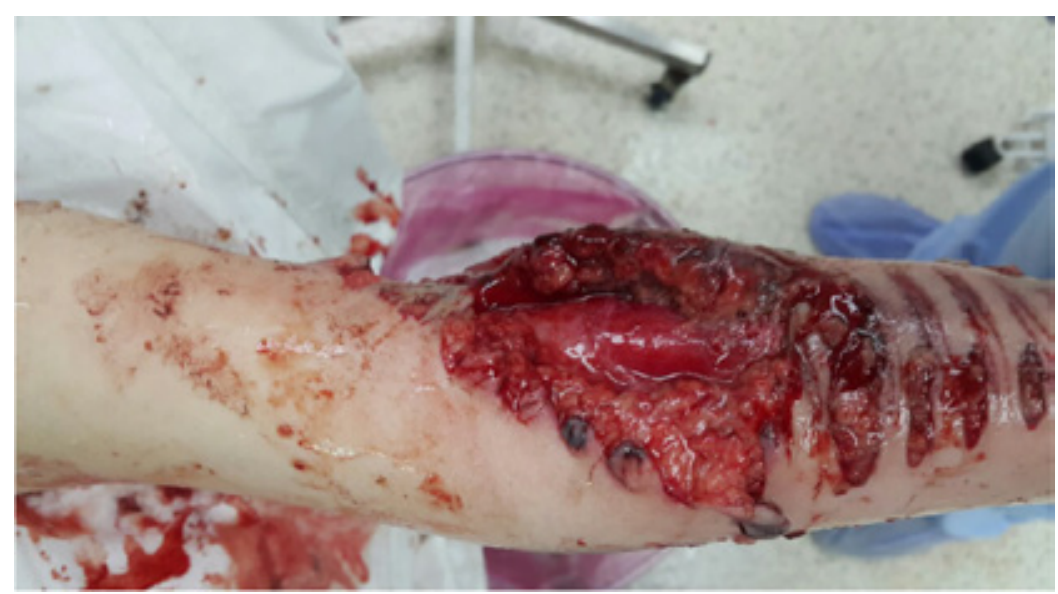

TABLA 1. Recomendaciones para padres y/o cuidadores

- Los niños pequeños deben ser supervisados adecuadamente, lo que incluye el agarre de la mano mientras viajan, en especial, al bajar las escaleras mecánicas.

- Todos los pasajeros deben tener precaución y permanecer alertas cuando suban a una escalera mecánica.

- Los niños no deben ser transportados en cochecitos de bebé mientras viajan en una escalera mecánica.

- Se debe enseñar a los niños a no correr, jugar o sentarse mientras suben o bajan en una escalera mecánica.

- Los niños deben mirar hacia adelante y sostenerse de los pasamanos.

- Los niños nunca deben sentarse en los escalones ni en los pasamanos de una escalera mecánica.

- Se debe informar a los niños que las escaleras mecánicas son peligrosas y no son lugares para saltar o jugar.

- Se debe prestar atención a los cordones de los calzados, ya que pueden ser agarrados por la escalera.

Adaptado y modificado por los autores de información de referencias. 2,4,10,11,12 
Las publicaciones mostraron que la parte del cuerpo más común lesionada en todas las edades fue la pierna, que representaba un tercio de todas las lesiones. ${ }^{3,4}$ Entre los niños menores de 5 años, la mano era el sitio de lesión más común (el 40,6\%), con lesiones frecuentes como resultado del atrapamiento $(\mathrm{el} 72,4 \%) .{ }^{4}$ En un reporte con más de 26000 casos, las amputaciones y avulsiones fueron poco frecuentes, pero el $71,4 \%$ (595 de 833) ocurrieron en menores de 5 años. ${ }^{4}$

La edad temprana con una marcha inestable, la supervisión inadecuada de un adulto que suele desestabilizar al menor aún más al acompañar la marcha traccionando de un brazo (sin mencionar las subluxaciones de codo por este motivo), la actividad inadecuada mientras viaja y los problemas mecánicos relacionados con la escalera mecánica aumentan el riesgo de lesiones. ${ }^{6}$ Los niños pequeños tienen mayores probabilidades de lesionarse debido a una caída mientras utilizan las escaleras mecánicas correctamente, en general, por falta de agarre a los pasamanos ubicados a excesiva altura para su talla, tal como sucedió con nuestra paciente. ${ }^{7}$

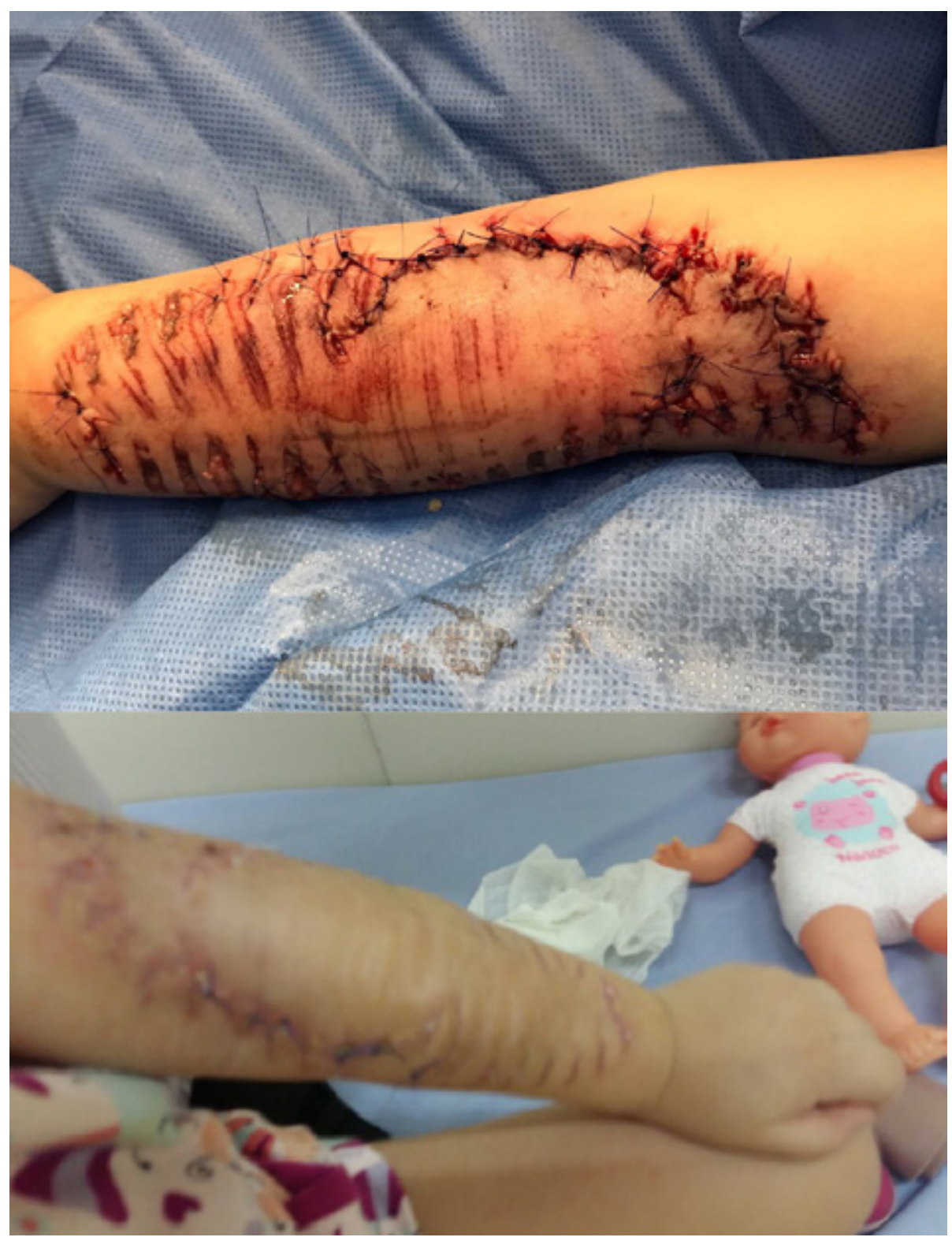


La lesionología con el uso de estos elementos nos indica que es necesaria la caída con carga de peso encima del área en contacto con el escalón ranurado para que la superficie de la piel no deslice y, por lo tanto, se incruste en el cuerpo. Las evaluaciones que se han hecho sobre la seguridad de estas escaleras muestran que el flujo de personas que sigue a aquel que trastabilla no puede evitar caer sobre el caído, por lo que descarga su peso hasta que se logra detener el movimiento de la banda transportadora. Los niños mayores se lesionan con más frecuencia por subir de forma incorrecta a la escalera mecánica, mientras juegan, corren o caminan. ${ }^{7}$ Los niños no deben ser transportados en vehículos de bebés en las escaleras mecánicas, debido a la posibilidad de caída por trabado del rodamiento. ${ }^{8,9}$

Dado que, en las publicaciones, ${ }^{3,4,6}$ se reporta la edad como un factor de riesgo, se considera que es necesario realizar mayor difusión de las medidas preventivas para concientizar a los padres. Con el uso de las medidas preventivas y los cuidados, la mayoría de estas lesiones pueden ser limitadas o completamente evitadas. ${ }^{2,4,10}$

Los pisos y obstáculos mojados deben limpiarse de manera oportuna y la señalización con reglas de seguridad debe colocarse en la entrada de cada escalera mecánica. ${ }^{11}$ Se deben seguir realizando esfuerzos para aumentar la conciencia sobre su uso seguro. Los carteles colocados en las entradas de la mayoría de las escaleras mecánicas como recordatorio de seguridad tienen una efectividad limitada porque, frecuentemente, no son leídos ni cumplidos. Por ello, algunas empresas recomiendan marcar la zona de seguridad en el escalón y poner señales de advertencia en la contrahuella. ${ }^{12}$ La Elevator Escalator Safety Foundation ${ }^{13}$ es una organización no gubernamental (ONG) que ofrece programas educativos que enseñan a niños y adultos cómo viajar correctamente en ascensores, escaleras mecánicas y pasillos móviles de manera segura.

Para finalizar, las lesiones relacionadas con escaleras mecánicas son poco frecuentes, pero pueden provocar lesiones traumáticas graves. En el caso expuesto, el fenómeno lacerante hubiera comprometido la vitalidad de la extremidad si el trauma se hubiera producido sobre la cara anterior del antebrazo donde se encuentran los dos pedículos arteriales en el antebrazo y la mano.
La morbilidad potencial asociada con cualquier incidente es demasiado alta para ignorarla. Se considera que es necesario realizar una mayor difusión de las medidas preventivas a fin de evitar la presentación de nuevos casos.

\section{Agradecimientos:}

Dra. Anahí Crocenzsi (equipo de Cirugía Plástica y Reparadora).

Dra. Verónica Sajewicz (Anestesiología, equipo de Cirugía Plástica y Reparadora).

Dra. Marisa Solange De Santis, Dr. Carmelo De Santis (Anestesiología, equipo de Traumatología y Ortopedia Infantil).

\section{REFERENCIAS}

1. Mitsubishi Electric. Datos históricos de las escaleras mecánicas. [Consulta: 2 de agosto de 2020]. Disponible en https://www.mitsubishielectric.com/elevator/es/ overview/e_m_walks/history.html.

2. McCann M, Zaleski N. Deaths and injuries involving elevators and escalators. 2006. [Consulta: 2 de agosto de 2020]. Disponible en http://www.elcosh.org/record/ document / 405/d000397.pdf.

3. Lim KB, Tey IK, Lokino ES, Yap RT, et al. Escalators, rubber clogs, and severe foot injuries in children. J Pediatr Orthop. 2010;30(5):414-9.

4. McGeehan J, Shields BJ, Wilkins JR 3rd, Ferketich AK, et al. Escalator-related injuries among children in the United States, 1990-2002. Pediatrics. 2006;118(2):e279-85.

5. McCann M, Zaleski N. Deaths and injuries involving elevators or escalators (revised). Centers for Disease Control and Prevention. [Consulta: 2 de agosto de 2020]. Disponible enhttps: / / www.cdc.gov/niosh/nioshtic-2/20039852.html.

6. Schminke LH, Jeger V, Evangelopoulos DS, Zimmerman $\mathrm{H}$, et al. Riding the Escalator: How Dangerous is it Really? West J Emerg Med. 2013;14(2):141-5.

7. Platt SL, Fine JS, Foltin GL. Escalator-related injuries in children. Pediatrics. 1997;100(2):E2.

8. Goh SS, Chong SL, Tyebally A. Danger in Shopping Centres - A Study on Escalator-Related Injuries in Children in Singapore. Ann Acad Med Singap. 2018;47(8):353-6.

9. Tripathi M, Tyebally A, Feng JX, Chong SL. A review of stroller-related and pram-related injuries to children in Singapore. Inj Prev. 2017;23(1):60-3.

10. Federación de Asociaciones y Cámaras de Ascensores de la República Argentina (FACARA). Las escaleras mecánicas y sus usos. [Consulta: 2 de agosto de 2020]. Disponible en: http: / / facara.com.ar/las-escaleras-mecanicas-y-sus-usos /.

11. XieK, LiuZ. Factors Influencing Escalator-Related Incidents in China: A Systematic Analysis Using ISM-DEMATEL Method. Int J Environ Res Public Health. 2019;16(14):2478.

12. Asai Marking System. Advertising \& Safety Alerts by Escalator Wrapping. [Consulta: 20 de agosto de 2020]. Disponible en https: / / www.ams-fleet.com/en/services / escalator/.

13. The Elevator Escalator Safety Foundation. Safe-T-Riders Kids Program. [Consulta: 20 de agosto de 2020]. Disponible en https: / / www.eesf.org/. 